\title{
MONOTONICITY FORMULAS OF EIGENVALUES AND ENERGY FUNCTIONALS ALONG THE RESCALED LIST'S EXTENDED RICCI FLOW
}

\author{
GUANGYUE HUANG AND ZHI LI
}

\begin{abstract}
In this paper, we study monotonicity formulas of eigenvalues and entropies along the rescaled List's extended Ricci flow. We derive some monotonicity formulas of eigenvalues of Laplacian which generalize those of $\mathrm{Li}$ in [8] and Cao-Hou-Ling in [3]. Moreover, we also consider monotonicity formulas of $\mathcal{F}_{k}$-functional which can be seen as a generalized $\mathcal{F}$-functional corresponding with steady Ricci breathers, and $\mathcal{W}_{k}$-functional which generalizes $\mathcal{W}$-functional corresponding with expanding Ricci breathers.
\end{abstract}

\section{INTRODUCTION}

Let $\left(M^{n}, g(t)\right)$ be a compact Riemannian manifold, $g(t)$ be a solution to the following List's extended Ricci flow which was introduced by B. List:

$$
\left\{\begin{array}{l}
\frac{\partial}{\partial t} g=-2 \operatorname{Ric}+2 \alpha d \varphi \otimes d \varphi \\
\varphi_{t}=\Delta \varphi
\end{array}\right.
$$

where $\alpha>0$ is a real constant, $\varphi=\varphi(t)$ is a smooth scalar function defined on $M^{n}$ and $\Delta$ denotes the Laplacian given by $g(t)$. When $\alpha=2$ and $\alpha=\frac{n-1}{n-2}$, the extended Ricci flow (1.1) have been studied by List in [6] and [7, respectively. Denote by $S_{i j}=R_{i j}-\alpha \varphi_{i} \varphi_{j}$ a symmetric two-tensor. Then (1.1) becomes

$$
\left\{\begin{array}{l}
\frac{\partial}{\partial t} g_{i j}=-2 S_{i j} \\
\varphi_{t}=\Delta \varphi
\end{array}\right.
$$

In this paper, we consider the following rescaled List's extended Ricci flow

$$
\left\{\begin{array}{l}
\frac{\partial}{\partial t} g_{i j}=-2\left(S_{i j}-\frac{r}{n} g_{i j}\right) \\
\varphi_{t}=\Delta \varphi
\end{array}\right.
$$

where $r=r(t)$ is a function depending only on $t$. In particular, (1.2) can be seen as a special case of (1.3) when $r=0$. On the other hand, if $r(t)=$ $\left(\int_{M} S d v\right) /\left(\int_{M} d v\right)$ (that is, $r$ is the average value of $S$ ), then (1.3) can be seen as the extended Hamilton normalized flow under the List's extended Ricci flow. Here

$$
S=g^{i j} S_{i j}=R-\alpha|\nabla \varphi|^{2}
$$

2000 Mathematics Subject Classification. 58C40, 53C44.

Key words and phrases. List's extended Ricci flow, eigenvalues, monotonicity.

The research of the first author was supported by NSFC No. 11371018, 11171091. 
is the trace of the two-tensor $S_{i j}$. For a constant $k \geq 1$, we defined the $\mathcal{F}_{k}$-functional as follows

$$
\mathcal{F}_{k}=\int_{M}\left(|\nabla f|^{2}+k S\right) e^{-f} d v
$$

If we define

$$
\lambda(g)=\inf _{f} \mathcal{F}_{k}
$$

where the infimum is taken over all smooth function $f$ which satisfies

$$
\int_{M} e^{-f} d v=1
$$

then the nondecreasing of the $\mathcal{F}_{k}$-functional implies the nondecreasing of $\lambda(g)$. In particular, $\lambda(g)$ defined in (1.6) is the lowest eigenvalue of the operator $-4 \Delta+k S$. In the first part, we consider eigenvalues of the operator

$$
-\Delta+b S
$$

with $b$ a constant. For $b=0$, we first derive the following evolution equation of eigenvalues on Laplacian under the rescaled List's extended Ricci flow (1.3). That is, we obtain

Theorem 1.1. Let $\lambda^{-\Delta}(t)$ be the eigenvalue of the operator $-\Delta$ corresponding to the normalized eigenfunction $u$, that is,

$$
-\Delta u=\lambda^{-\Delta} u, \quad \int_{M} u^{2} d v=1 .
$$

Then under the rescaled List's extended Ricci flow (1.3),

$$
\frac{d}{d t} \lambda^{-\Delta}=-\frac{2 r}{n} \lambda^{-\Delta}+\int_{M}\left(\lambda^{-\Delta} S u^{2}-S|\nabla u|^{2}+2 S^{i j} u_{i} u_{j}\right) d v
$$

Theorem 1.2. Let $\lambda^{-\Delta+\frac{1}{2} S}(t)$ be the eigenvalue of the operator $-\Delta+\frac{1}{2} S$ corresponding to the normalized eigenfunction $u$, that is,

$$
\left(-\Delta+\frac{1}{2} S\right) u=\lambda^{-\Delta+\frac{1}{2} S} u, \quad \int_{M} u^{2} d v=1 .
$$

Then under the rescaled List's extended Ricci flow (1.3),

$$
\frac{d}{d t} \lambda^{-\Delta+\frac{1}{2} S}=-\frac{2 r}{n} \lambda^{-\Delta+\frac{1}{2} S}+\int_{M}\left[\left|S_{i j}\right|^{2} u^{2}+2 S^{i j} u_{i} u_{j}+\alpha(\Delta \varphi)^{2} u^{2}\right] d v
$$


Moreover, if $S_{i j}(t) \geq 0$ for all $t$, the eigenvalues of the operator $-\Delta+\frac{1}{2} S$ satisfy

$$
\begin{aligned}
\frac{d}{d t}\left(\lambda^{-\Delta+\frac{1}{2} S} e^{\frac{2}{n} \int_{0}^{t} r(s) d s}\right)= & e^{\frac{2}{n} \int_{0}^{t} r(s) d s}\left\{\int _ { M } \left[\left|S_{i j}\right|^{2} u^{2}+2 S^{i j} u_{i} u_{j}\right.\right. \\
& \left.\left.+\alpha(\Delta \varphi)^{2} u^{2}\right] d v\right\} \geq 0
\end{aligned}
$$

and $\lambda^{-\Delta+\frac{1}{2} S} e^{\frac{2}{n} \int_{0}^{t} r(s) d s}$ is nondecreasing under the rescaled List's extended Ricci flow (1.3). Furthermore, the monotonicity is strict unless the metric is Ricci flat.

It is well-known that, under the List's extended Ricci flow (1.2), the nonnegativity of $S$ is preserved. In this paper, we will prove that the nonnegativity of $S$ is also preserved under the rescaled List's extended Ricci flow (1.3) for all $r(t)$. That is,

Theorem 1.3. The nonnegativity of $S$ is preserved under the rescaled List's extended Ricci flow (1.3).

In order to state the following results on eigenvalues, we first introduce the following definition:

$$
S_{\min }(0)=\min _{x \in M} S(x, 0) .
$$

By virtue of Theorem 1.3, we prove the following

Theorem 1.4. Let $(g(t), \varphi(t))$ be a solution to the rescaled List's extended Ricci flow (1.3) with $S_{i j}(t) \geq \theta g_{i j}(t)$ holding for some $\theta \geq \frac{1}{2}$. Let $\lambda^{-\Delta}(t)$ be the eigenvalue of the operator $-\Delta$.

(1) If $S_{\min }(0) \geq 0$, then $\lambda^{-\Delta} e^{\frac{2}{n} \int_{0}^{t} r(s) d s}$ is nondecreasing along the rescaled List's extended Ricci flow (1.3).

(2) For all t, we have

$$
\frac{d}{d t} \ln \left(\lambda^{-\Delta} e^{\frac{2}{n} \int_{0}^{t} r(s) d s}\right) \geq 2 \theta x(t) .
$$

Moreover, $\lambda(t)$ has the lower bound

$$
\lambda^{-\Delta}(t) e^{\frac{2}{n} \int_{0}^{t} r(s) d s} \geq \lambda(0) e^{2 \theta \int_{0}^{t} x(s) d s}
$$

depending only on $t$, where

$$
x(t)=\frac{S_{\min }(0) e^{-\frac{2}{n} \int_{0}^{t} r(s) d s}}{1-\frac{2}{n} S_{\min }(0) \int_{0}^{t}\left(e^{-\frac{2}{n} \int_{0}^{s} r(\tilde{s}) d \tilde{s}}\right) d s} .
$$

In particular, for compact Riemannian surfaces, we obtain the following consequences from Theorem 1.4 
Corollary 1.5. Let $(g(t), \varphi(t))$ be a solution to the rescaled List's extended Ricci flow (1.3) on $M^{2}$. Let $\lambda^{-\Delta}(t)$ be the eigenvalue of the operator $-\Delta$.

(i) If

$$
R_{i j} \leq \epsilon u_{i} u_{j}
$$

where $\epsilon \leq \frac{2 \alpha(\theta-1)}{2 \theta-1}$ with $\theta>\frac{1}{2}$, then the following holds:

(1) If $S_{\min }(0) \geq 0$, then $\lambda^{-\Delta} e_{0}^{t} r(s) d s$ is nondecreasing along the rescaled List's extended Ricci flow (1.3).

(2) For all t, we have

$$
\frac{d}{d t} \ln \left(\lambda^{-\Delta} e^{\int_{0}^{t} r(s) d s}\right) \geq 2 \theta x(t) .
$$

Moreover, $\lambda(t)$ has the lower bound

$$
\lambda^{-\Delta}(t) e^{\int_{0}^{t} r(s) d s} \geq \lambda(0) e^{2 \theta \int_{0}^{t} x(s) d s},
$$

depending only on $t$, where

$$
x(t)=\frac{S_{\min }(0) e^{-\int_{0}^{t} r(s) d s}}{1-S_{\min }(0) \int_{0}^{t}\left(e^{-\int_{0}^{s} r(\tilde{s}) d \tilde{s}}\right) d s} .
$$

(ii) If $|\nabla \varphi|^{2} g_{i j} \geq 2 \varphi_{i} \varphi_{j}$, then the following holds:

(1) If $S_{\min }(0) \geq 0$, then $\lambda^{-\Delta} e_{0}^{t} r(s) d s$ is nondecreasing along the rescaled List's extended Ricci flow (1.3).

(2) For all t, we have

$$
\frac{d}{d t} \ln \left(\lambda^{-\Delta} e^{\int_{0}^{t} r(s) d s}\right) \geq x(t) .
$$

Moreover, $\lambda(t)$ has the lower bound

$$
\lambda^{-\Delta}(t) e^{\int_{0}^{t} r(s) d s} \geq \lambda(0) e^{\int_{0}^{t} x(s) d s}
$$

depending only on $t$, where

$$
x(t)=\frac{S_{\min }(0) e^{-\int_{0}^{t} r(s) d s}}{1-S_{\min }(0) \int_{0}^{t}\left(e^{-\int_{0}^{s} r(\tilde{s}) d \tilde{s}}\right) d s} .
$$

Remark 1.1. It should be pointed out that for $r=0$ and $\alpha=2$, our above results on eigenvalues reduce to the corresponding results of $\mathrm{Li}$ in [8]. In particular, our Theorem 1.3 is new.

Remark 1.2. Some related results for monotonicity formulas of eigenvalues on Laplacian along the Ricci flow, we refer to [1,2,10] and among others [5] for later development.

Next, we study monotonicity formulas of eigenvalues on Laplacian on Riemannian surfaces. We obtain the following results: 
Theorem 1.6. Let $\lambda^{-\Delta+b S}(t)$ be the eigenvalue of the operator $-\Delta+b S$ with normalized eigenfunction $u$ on $M^{2}$ with $S(t) \geq 0$ holding for all $t$, that is,

$$
(-\Delta+b S) u=\lambda^{-\Delta+b S} u, \quad \int_{M} u^{2} d v=1 .
$$

Then if $|\nabla \varphi|^{2} g_{i j} \geq 2 \varphi_{i} \varphi_{j}$ and $0<b \leq \frac{1}{2}$, we have

$$
\begin{aligned}
\frac{d}{d t}\left(\lambda^{-\Delta+b S} e^{\int_{0}^{t} r(s) d s}\right) \geq & e^{\int_{0}^{t} r(s) d s}\left\{\int _ { M } \left\{2 b^{2} S^{2} u^{2}+(1-2 b) \lambda S u^{2}+2 b S|\nabla u|^{2}\right.\right. \\
& \left.\left.\left.+b \alpha^{2}|\nabla \varphi|^{4} u^{2}+2 b \alpha(\Delta \varphi)^{2} u^{2}\right]\right\} d v\right\} \\
& \geq 0
\end{aligned}
$$

We also obtain the following bounds for eigenvalues of the operator $-\Delta+$ $b S$ on compact Riemannian surfaces.

Theorem 1.7. Let $\lambda^{-\Delta+b S}(t)$ be the eigenvalue of the operator $-\Delta+b S$ on $M^{2}$. If $|\nabla \varphi|^{2} g_{i j} \geq 2 \varphi_{i} \varphi_{j}$ and $0<b \leq \frac{1}{2}$, we have

$$
\left[1-t S_{\min }(0)\right] \lambda-\frac{b^{2} S_{\min }^{2}(0)}{2} \ln \left[1-t S_{\min }(0)\right]
$$

is nondecreasing under the List's extended Ricci flow (1.2). Moreover, $\lambda(t)$ has the lower bound

$$
\lambda(t) \geq \frac{1}{1-t S_{\min }(0)} \lambda(0)+\frac{b^{2} S_{\min }(0)}{2\left[1-t S_{\min }(0)\right]} \ln \left[1-t S_{\min }(0)\right]
$$

depending only on $t$.

Theorem 1.8. Let $\lambda^{-\Delta+b S}(t)$ be the eigenvalue of the operator $-\Delta+b S$ on $M^{2}$ with $r>0$ and $S_{\min }(0)>0$. If $|\nabla \varphi|^{2} g_{i j} \geq 2 \varphi_{i} \varphi_{j}$ and $0<b \leq \frac{1}{2}$, we have

$$
\frac{d}{d t}(\ln \lambda) \geq \frac{S_{\min }(0) e^{-\int_{0}^{t} r(s) d s}}{1-S_{\min }(0) \int_{0}^{t}\left(e^{-\int_{0}^{s} r(\tilde{s}) d \tilde{s}}\right) d s}-r
$$

under the rescaled List's extended Ricci flow (1.3). Moreover, $\lambda(t)$ has the lower bound

$$
\lambda(t) \geq \lambda(0) e^{\int_{0}^{t} \tilde{x}(s) d s},
$$

depending only on $t$, where

$$
\tilde{x}(t)=\frac{S_{\min }(0) e^{-\int_{0}^{t} r(s) d s}}{1-S_{\min }(0) \int_{0}^{t}\left(e^{-\int_{0}^{s} r(\tilde{s}) d \tilde{s}}\right) d s}-r .
$$


Remark 1.3. When $r=0$, Theorem 1.6 becomes Theorem 1.6 of Cao-HouLing in [3]. When $\varphi=0$ and $r(t)=\left(\int_{M} R d v\right) /\left(\int_{M} d v\right)$, our Theorems 1.7. 1.8 reduce to Theorem 3.4 and Theorem 3.3 of Cao-Hou-Ling in [3], respectively.

In the rest of this paper, we consider monotonicity formulas of $\mathcal{F}_{k}$-functional which can be seen as a generalized $\mathcal{F}$-functional corresponding with steady Ricci breathers, and $\mathcal{W}_{k}$-functional which can be seen as a generalized $\mathcal{W}$ functional corresponding with expanding Ricci breathers. Under the following evolution equation

$$
\left\{\begin{array}{l}
\frac{\partial}{\partial t} g_{i j}=-2\left(S_{i j}-\frac{r}{n} g_{i j}\right) \\
\varphi_{t}=\Delta \varphi \\
f_{t}=-\Delta f+|\nabla f|^{2}-S+r
\end{array}\right.
$$

we proved the following results:

Theorem 1.9. Under the system (1.23), we have

$$
\begin{aligned}
\frac{d}{d t} \mathcal{F}_{k}= & -\frac{2 r}{n} \mathcal{F}_{k}+2(k-1) \int_{M}\left(\left|S_{i j}\right|^{2}+\alpha(\Delta \varphi)^{2}\right) e^{-f} d v \\
& +2 \int_{M}\left(\left|S_{i j}+f_{i j}\right|^{2}+\alpha|\Delta \varphi-\langle\nabla f, \nabla \varphi\rangle|^{2}\right) e^{-f} d v,
\end{aligned}
$$

or equivalently,

$$
\begin{aligned}
\frac{d}{d t} \mathcal{F}_{k}= & \frac{2 r}{n}\left(\mathcal{F}_{k}-k r\right)+2(k-1) \int_{M}\left(\left|S_{i j}-\frac{r}{n} g_{i j}\right|^{2}+\alpha(\Delta \varphi)^{2}\right) e^{-f} d v \\
& +2 \int_{M}\left(\left|S_{i j}+f_{i j}-\frac{r}{n} g_{i j}\right|^{2}+\alpha|\Delta \varphi-\langle\nabla f, \nabla \varphi\rangle|^{2}\right) e^{-f} d v
\end{aligned}
$$

Remark 1.4. It was pointed out that for $\alpha=2$ and $k=1$, List [6] and Müller [11] studied the monotonicity of $\mathcal{F}_{k}$-functional under the List's extended Ricci flow (1.2). Later, In [8], Li studied the monotonicity of $\mathcal{F}_{k}$-functional for $\alpha=2$ and all $k \geq 1$ under (1.2). In particular, when $\varphi=0$ and $k=1$, the $\mathcal{F}_{k}$-functional (1.5) becomes the Perelman's $\mathcal{F}$-functional.

Applying (1.25) in Theorem 1.9, we can also obtain the following result:

Theorem 1.10. Let $\left(M^{n}, g(t)\right)$ be a compact Riemannian manifold with $g(t)$ satisfying the rescaled List's extended Ricci flow (1.3). We let $\lambda(t)$ be the lowest eigenvalue of the operator $-4 \Delta+k S$ with $k \geq 1$. If the average value of $S$ is nonnegative for all $t$, then $\lambda(t)$ is nondecreasing along (1.3). Moreover, the monotonicity is strict unless the metric is Einstein.

As a direct application of (1.24) in Theorem 1.9, we can obtain the following results: 
Corollary 1.11. Under the system (1.23) and $k \geq 1$, we have

$$
\begin{aligned}
\frac{d}{d t}\left(\mathcal{F}_{k} e^{\frac{2}{n} \int_{0}^{t} r(s) d s}\right)= & e^{\frac{2}{n} \int_{0}^{t} r(s) d s}\left\{2(k-1) \int_{M}\left(\left|S_{i j}\right|^{2}+\alpha(\Delta \varphi)^{2}\right) e^{-f} d v\right. \\
& \left.+2 \int_{M}\left(\left|S_{i j}+f_{i j}\right|^{2}+\alpha|\Delta \varphi-\langle\nabla f, \nabla \varphi\rangle|^{2}\right) e^{-f} d v\right\} \\
\geq & 0,
\end{aligned}
$$

which shows that $\mathcal{F}_{k} e^{\frac{2}{n} \int_{0}^{t} r(s) d s}$ is nondecreasing along (1.23). Moreover, the monotonicity is strict unless the metric is Ricci flat.

Remark 1.5. Choosing $r=0$ and $\alpha=2$, then (1.24) reduces the formula (1-6) in Theorem 1.1 of $\mathrm{Li}$ [8]. On the other hand, under the normalized Ricci flow of Hamilton, Li in [9] also obtained a similar result as Theorem 1.10 .

As in [8], for a constant $k \geq 1$, we define the following $\mathcal{W}_{k}$-functional:

$$
\mathcal{W}_{k}=\tau^{2} \int_{M}\left[k\left(S+\frac{n}{2 \tau}\right)+|\nabla f|^{2}\right] e^{-f} d v
$$

Under the following coupled system

$$
\left\{\begin{array}{l}
\frac{\partial}{\partial t} g_{i j}=-2\left(S_{i j}-\frac{r}{n} g_{i j}\right), \\
\varphi_{t}=\Delta \varphi \\
f_{t}=-\Delta f+|\nabla f|^{2}-S+r \\
\tau_{t}=1
\end{array}\right.
$$

we obtain the following results:

Theorem 1.12. Under the system (1.28) and $k \geq 1$, we have

$$
\begin{aligned}
\frac{d}{d t} \mathcal{W}_{k}= & 2 \tau^{2}\left\{-\frac{r}{n} \mathcal{F}_{k}+(k-1) \int_{M}\left(\left|S_{i j}+\frac{1}{2 \tau} g_{i j}\right|^{2}+\alpha(\Delta \varphi)^{2}\right) e^{-f} d v\right. \\
& \left.+\int_{M}\left(\left|S_{i j}+f_{i j}+\frac{1}{2 \tau} g_{i j}\right|^{2}+\alpha|\Delta \varphi-\langle\nabla f, \nabla \varphi\rangle|^{2}\right) e^{-f} d v\right\},
\end{aligned}
$$


or equivalently,

$$
\begin{aligned}
\frac{d}{d t} \mathcal{W}_{k}= & 2 \tau^{2}\left\{\frac{r}{n}\left(\mathcal{F}_{k}-k r\right)+\frac{k r}{\tau}\right. \\
& +(k-1) \int_{M}\left(\left|S_{i j}+\frac{1}{2 \tau} g_{i j}-\frac{r}{n} g_{i j}\right|^{2}+\alpha(\Delta \varphi)^{2}\right) e^{-f} d v \\
& \left.+\int_{M}\left(\left|S_{i j}+f_{i j}+\frac{1}{2 \tau} g_{i j}-\frac{r}{n} g_{i j}\right|^{2}+\alpha|\Delta \varphi-\langle\nabla f, \nabla \varphi\rangle|^{2}\right) e^{-f} d v\right\} .
\end{aligned}
$$

As a direct application of the formula (1.29), we obtain

Corollary 1.13. Under the system (1.28) and $k \geq 1$, we have

$$
\begin{aligned}
\frac{d}{d t} \mathcal{W}_{k}+\frac{2 r}{n} \tau^{2} \mathcal{F}_{k}= & 2 \tau^{2}\left\{(k-1) \int_{M}\left(\left|S_{i j}+\frac{1}{2 \tau} g_{i j}\right|^{2}+\alpha(\Delta \varphi)^{2}\right) e^{-f} d v\right. \\
& \left.+\int_{M}\left(\left|S_{i j}+f_{i j}+\frac{1}{2 \tau} g_{i j}\right|^{2}+\alpha|\Delta \varphi-\langle\nabla f, \nabla \varphi\rangle|^{2}\right) e^{-f} d v\right\} \\
\geq 0 &
\end{aligned}
$$

Moreover, the equality in (1.31) holds if and only if the metric is Einstein.

Remark 1.6. When $r=0$ and $\alpha=2$, then (1.29) reduces the formula (1-8) in Theorem 1.3 of $\mathrm{Li}[8]$.

This paper is organized as follows: In Section two, we study monotonicity formulas for eigenvalues of the operator $-\Delta+b S$. We mainly consider monotonicity formulas for eigenvalues of the Laplacian under the rescaled List's extended Ricci flow (1.3) and give some dependent lower bounds of eigenvalues. Moreover, Theorem 1.1 Theorem 1.4 have been proved in this part. In Section three, we deal with monotonicity formulas for eigenvalues of the operator $-\Delta+b S$ with $0<b \leq \frac{1}{2}$ on compact Riemannian surfaces and obtain some interesting results. In this part, we give proofs of Theorem 1.6. Theorem 1.8. In the last Section four, we study the first variations of $\mathcal{F}_{k}$-functional and $\mathcal{W}_{k}$-functional. These functionals are very important to study entropies corresponding to the List's extended Ricci flow (1.2). In this part, Theorem 1.9-Theorem 1.12 have been proved.

\section{Proof of Theorem 1.1-Theorem 1.4}

We first give a Lemma which will be used later. 
Lemma 2.1. Under the rescaled List's extended Ricci flow (1.3), we have

$$
\begin{gathered}
\frac{\partial}{\partial t} g^{i j}=2 g^{i k} g^{j l}\left(S_{k l}-\frac{r}{n} g_{k l}\right), \\
(d v)_{t}=(-S+r) d v, \\
S_{t}=\Delta S+2\left|S_{i j}\right|^{2}-\frac{2 r}{n} S+2 \alpha(\Delta \varphi)^{2} .
\end{gathered}
$$

In particular, when $r(t)=\left(\int_{M} S d v\right) /\left(\int_{M} d v\right)$ (that is, $r$ is the average value of $S)$, then (2.2) shows that the volume of $(M, g(t))$ is a constant for all $t$.

Proof. It has been shown in [6] (see Lemma 1.4 in [6]) that if the metric $g(t)$ satisfies

$$
\frac{\partial}{\partial t} g_{i j}=v_{i j}
$$

where $v_{i j}$ is a symmetric two tensor, then

$$
\begin{gathered}
\frac{\partial}{\partial t} g^{i j}=-v^{i j} \\
(d v)_{t}=\frac{1}{2}\left(\operatorname{tr}_{\mathrm{g}} v\right) d v
\end{gathered}
$$

with $\operatorname{tr}_{\mathrm{g}} v$ denoting the trace of $v_{i j}$ with respect to $g$, and

$$
R_{t}=-\Delta\left(\operatorname{tr}_{\mathrm{g}} v\right)+g^{k j} g^{i l} v_{i j, k l}-g^{k j} g^{i l} R_{k l} v_{i j}
$$

Hence, inserting $v_{i j}=-2\left(S_{i j}-\frac{r}{n} g_{i j}\right), \operatorname{tr}_{\mathrm{g}} v=-2(S-r)$ into (2.5) and (2.6), we obtain (2.1) and (2.2), respectively.

Next, we prove (2.3). In fact, from (2.7), we have

$$
\begin{aligned}
R_{t} & =2 \Delta S-2 g^{k j} g^{i l} S_{i j, k l}+2 g^{k j} g^{i l} R_{k l}\left(S_{i j}-\frac{r}{n} g_{i j}\right) \\
& =2 \Delta S-2 g^{k j} g^{i l} S_{i j, k l}+2 g^{k j} g^{i l}\left(S_{k l}+\alpha \varphi_{k} \varphi_{l}\right)\left(S_{i j}-\frac{r}{n} g_{i j}\right) \\
& =2 \Delta S-2 g^{k j} g^{i l} S_{i j, k l}+2\left|S_{i j}\right|^{2}-\frac{2 r}{n} S+2 \alpha S_{i j} \varphi^{i} \varphi^{j}-\alpha \frac{2 r}{n}|\nabla \varphi|^{2} .
\end{aligned}
$$

By the definition of $S_{i j}$ and the contracted Bianchi indentity, we also have

$$
\begin{aligned}
g^{k j} S_{i j, k} & =g^{k j} R_{i j, k}-g^{k j} \alpha\left(\varphi_{i} \varphi_{j}\right)_{, k} \\
& =\frac{1}{2}\left(R-\alpha|\nabla \varphi|^{2}\right)_{, i}-\alpha(\Delta \varphi) \varphi_{i} \\
& =\frac{1}{2} S_{, i}-\alpha(\Delta \varphi) \varphi_{i}
\end{aligned}
$$

Thus, (2.8) becomes

$$
\begin{aligned}
R_{t}= & \Delta S+2\left|S_{i j}\right|^{2}-\frac{2 r}{n} S+2 \alpha S_{i j} \varphi^{i} \varphi^{j}-\alpha \frac{2 r}{n}|\nabla \varphi|^{2}+2 \alpha(\Delta \varphi)^{2} \\
& +2 \alpha\langle\nabla \Delta \varphi, \nabla \varphi\rangle .
\end{aligned}
$$

It follows that

$$
\begin{aligned}
S_{t} & =\left(R-\alpha|\nabla \varphi|^{2}\right)_{t} \\
& =\Delta S+2\left|S_{i j}\right|^{2}-\frac{2 r}{n} S+2 \alpha(\Delta \varphi)^{2}
\end{aligned}
$$


and the desired (2.3) follows. We complete the proof of Lemma 2.1.

Let $u$ be the eigenfunction corresponding to eigenvalue $\lambda$ of the operator $-\Delta+b S$, that is,

$$
(-\Delta+b S) u=\lambda u \text {. }
$$

Multiplying both sides of (2.12) with $u$ and integrating on $M$, we have

$$
\lambda=\int_{M}\left(|\nabla u|^{2}+b S u^{2}\right) d v
$$

Using Lemma 2.1, we have

$$
\begin{aligned}
\frac{d}{d t} \lambda= & \int_{M}\left(2 u_{i} u_{j} \frac{\partial}{\partial t} g^{i j}+2\left(u_{t}\right)^{i} u_{i}+b S_{t} u^{2}+2 b S u u_{t}\right) d v \\
& +\int_{M}\left(|\nabla u|^{2}+b S u^{2}\right)(-S+r) d v \\
= & \int_{M}\left(2 S^{i j} u_{i} u_{j}-\frac{2 r}{n}|\nabla u|^{2}-2 u_{t} \Delta u+b S_{t} u^{2}+2 b S u u_{t}\right) d v \\
& +\int_{M}\left(|\nabla u|^{2}+b S u^{2}\right)(-S+r) d v .
\end{aligned}
$$

From (2.9) and the Stokes formula, we have

$$
2 \int_{M} S^{i j} u_{i} u_{j} d v=\int_{M}\left(-S_{, i} u^{i} u-2 S^{i j} u_{i j} u+2 \alpha(\Delta \varphi)\langle\nabla u, \nabla \varphi\rangle u\right) d v .
$$

On the other hand,

$$
-\int_{M}|\nabla u|^{2} S d v=\int_{M}\left(S \Delta u+S_{, i} u^{i}\right) u d v
$$


MONOTONICITY FORMULAS OF EIGENVALUES AND ENERGY FUNCTIONALS 11 Inserting (2.15) and (2.16) into (2.14) yields

$$
\begin{aligned}
\frac{d}{d t} \lambda= & \int_{M}\left\{-\frac{2 r}{n}|\nabla u|^{2}+b S_{t} u^{2}-2 S^{i j} u_{i j} u+2 \alpha(\Delta \varphi)\langle\nabla u, \nabla \varphi\rangle u\right. \\
& \left.+2 u_{t}(-\Delta u+b S u)-S u(-\Delta u+b S u)+r\left(|\nabla u|^{2}+b S u^{2}\right)\right\} d v \\
= & \int_{M}\left\{-\frac{2 r}{n}|\nabla u|^{2}+b S_{t} u^{2}-2 S^{i j} u_{i j} u+2 \alpha(\Delta \varphi)\langle\nabla u, \nabla \varphi\rangle u\right. \\
& \left.+\lambda 2 u_{t} u+\lambda(-S+r) u^{2}\right\} d v \\
= & \int_{M}\left\{-\frac{2 r}{n}|\nabla u|^{2}+b S_{t} u^{2}-2 S^{i j} u_{i j} u+2 \alpha(\Delta \varphi)\langle\nabla u, \nabla \varphi\rangle u\right\} \\
& +\lambda\left(\int_{M} u^{2} d v\right)_{t} \\
= & \int_{M}\left\{-\frac{2 r}{n}|\nabla u|^{2}+b S_{t} u^{2}-2 S^{i j} u_{i j} u+2 \alpha(\Delta \varphi)\langle\nabla u, \nabla \varphi\rangle u\right\} d v .
\end{aligned}
$$

Applying (2.3) in Lemma 2.1 into (2.17) yields

$$
\begin{aligned}
\frac{d}{d t} \lambda= & \int_{M}\left\{-\frac{2 r}{n}\left(|\nabla u|^{2}+b S u^{2}\right)+2 b\left|S_{i j}\right|^{2} u^{2}-2 S^{i j} u_{i j} u+b u^{2}(\Delta S)\right. \\
& \left.+2 b \alpha(\Delta \varphi)^{2} u^{2}+2 \alpha(\Delta \varphi)\langle\nabla u, \nabla \varphi\rangle u\right\} d v \\
= & -\frac{2 r}{n} \lambda+\int_{M}\left\{2 b\left|S_{i j}\right|^{2} u^{2}-2 S^{i j} u_{i j} u+b u^{2} \Delta S\right. \\
& \left.+2 b \alpha(\Delta \varphi)^{2} u^{2}+2 \alpha(\Delta \varphi)\langle\nabla u, \nabla \varphi\rangle u\right\} d v .
\end{aligned}
$$

Using the Stokes formula again, we have

$$
\begin{aligned}
-2 \int_{M} S^{i j} u_{i j} u d v & =\int_{M}\left(2 S^{i j}{ }_{j} u_{i} u d v+2 S^{i j} u_{i} u_{j}\right) d v \\
& =\int_{M}\left[S_{, i} u_{i} u+2 S^{i j} u_{i} u_{j}-2 \alpha(\Delta \varphi)\langle\nabla u, \nabla \varphi\rangle u\right] d v \\
& =\int_{M}\left[-\frac{1}{2} u^{2} \Delta S+2 S^{i j} u_{i} u_{j}-2 \alpha(\Delta \varphi)\langle\nabla u, \nabla \varphi\rangle u\right] d v .
\end{aligned}
$$


Therefore, (2.18) becomes

$$
\begin{aligned}
\frac{d}{d t} \lambda= & -\frac{2 r}{n} \lambda+\int_{M}\left\{2 b\left|S_{i j}\right|^{2} u^{2}+2 S^{i j} u_{i} u_{j}+\left(b-\frac{1}{2}\right) u^{2} \Delta S+2 b \alpha(\Delta \varphi)^{2} u^{2}\right\} d v \\
= & -\frac{2 r}{n} \lambda+\int_{M}\left\{2 b\left|S_{i j}\right|^{2} u^{2}+2 S^{i j} u_{i} u_{j}+2 b \alpha(\Delta \varphi)^{2} u^{2}\right. \\
& \left.+(2 b-1) S\left[(b S-\lambda) u^{2}+|\nabla u|^{2}\right]\right\} d v .
\end{aligned}
$$

Hence, the following consequence follows:

Proposition 2.2. Let $\lambda^{-\Delta+b S}(t)$ be the eigenvalue of the operator $-\Delta+b S$ corresponding to the normalized eigenfunction $u$, that is,

$$
(-\Delta+b S) u=\lambda^{-\Delta+b S} u, \quad \int_{M} u^{2} d v=1 .
$$

Then

$$
\begin{aligned}
\frac{d}{d t} \lambda^{-\Delta+b S}= & -\frac{2 r}{n} \lambda^{-\Delta+b S}+\int_{M}\left\{2 b\left|S_{i j}\right|^{2} u^{2}+2 S^{i j} u_{i} u_{j}+2 b \alpha(\Delta \varphi)^{2} u^{2}\right. \\
& \left.+(2 b-1) S\left[(b S-\lambda) u^{2}+|\nabla u|^{2}\right]\right\} d v
\end{aligned}
$$

Proof of Theorems 1.1 and 1.2, From Proposition 2.2, it is easy to derive Theorem 1.1 by letting $b=0$ and Theorem 1.2 by letting $b=\frac{1}{2}$, respectively.

Next, we give the proof of Theorem 1.3 by the Lemma 2.12 in [4] (see Page 99 in 4]).

Proof of Theorem 1.3. Using the Cauchy inequality

$$
\left|S_{i j}\right|^{2} \geq \frac{1}{n} S^{2}
$$

we obtain from (2.3)

$$
S_{t} \geq \Delta S+\frac{2}{n} S^{2}-\frac{2 r}{n} S
$$

Comparing $S$ with the corresponding solution of ODE

$$
\frac{d}{d t} x=\frac{2}{n} x^{2}-\frac{2 r}{n} x, \quad x(0)=S_{\min }(0)
$$

gives

$$
S(x, t) \geq x(t):=\frac{S_{\min }(0) e^{-\frac{2}{n} \int_{0}^{t} r(s) d s}}{1-\frac{2}{n} S_{\min }(0) \int_{0}^{t}\left(e^{-\frac{2}{n} \int_{0}^{s} r(\tilde{s}) d \tilde{s}}\right) d s}
$$


where $x(t)$ is the solution of (2.23). In particular, when $S_{\min }(0)=0$, then $S(0) \geq 0$ and (2.24) gives

$$
S(x, t) \geq 0
$$

for all $t$. The desired Theorem 1.3 is attained.

Proof of Theorem 1.4. From (2.21), we have

$$
\begin{aligned}
\frac{d}{d t}\left(\lambda^{-\Delta} e^{\frac{2}{n} \int_{0}^{t} r(s) d s}\right) & =e^{\frac{2}{n} \int_{0}^{t} r(s) d s}\left\{\int_{M}\left(\lambda^{-\Delta} S u^{2}-S|\nabla u|^{2}+2 S^{i j} u_{i} u_{j}\right) d v\right\} \\
& \geq e^{\frac{2}{n} \int_{0}^{t} r(s) d s}\left\{\lambda^{-\Delta} \int_{M} S u^{2} d v+(2 \theta-1) \int_{M} S|\nabla u|^{2} d v\right\}
\end{aligned}
$$

which shows (1) holds. On the other hand, applying (2.24) into (2.26), we achieve

$$
\begin{aligned}
\frac{d}{d t}\left(\lambda^{-\Delta} e^{\frac{2}{n} \int_{0}^{t} r(s) d s}\right) & \geq e^{\frac{2}{n} \int_{0}^{t} r(s) d s}\left\{\lambda^{-\Delta} x(t)+(2 \theta-1) x(t) \lambda^{-\Delta}\right\} \\
& =2 \theta e^{\frac{2}{n} \int_{0}^{t} r(s) d s} x(t) \lambda^{-\Delta}
\end{aligned}
$$

which shows (2) holds.

Proof of Corollary 1.5. As in [8] of Li, using the fact $R_{i j}=\frac{R}{2} g_{i j}$, we can compute

$$
\begin{aligned}
S_{i j} V^{i} V^{j} & =\left(\frac{R}{2} g_{i j}-\alpha \varphi_{i} \varphi_{j}\right) V^{i} V^{j} \\
& =\frac{R}{2}|V|^{2}-\alpha\langle\nabla \varphi, V\rangle^{2} \\
& \geq \frac{R}{2}|V|^{2}-\alpha|\nabla \varphi|^{2}|V|^{2} \\
& \geq\left(\frac{R}{2}-\alpha|\nabla \varphi|^{2}\right)|V|^{2}
\end{aligned}
$$

where $V=\left(V^{i}\right)$. Since $R_{i j} \leq \epsilon u_{i} u_{j}$ and $\epsilon \leq \frac{2 \alpha(\theta-1)}{2 \theta-1}$ with $\theta>\frac{1}{2}$, we have

$$
\left(\frac{1}{2}-\theta\right) R+(\theta-1) \alpha|\nabla \varphi|^{2} \geq 0
$$

which is equivalent to

$$
\left(\frac{R}{2}-\alpha|\nabla \varphi|^{2}\right)|V|^{2} \geq \theta S|V|^{2}
$$

Therefore, we have $S_{i j} \geq \theta S g_{i j}$ from (2.28) and the consequence (i) follows. 
On the other hand, we can check that if $|\nabla \varphi|^{2} g_{i j} \geq 2 \varphi_{i} \varphi_{j}$, then

$$
\begin{aligned}
S_{i j} V^{i} V^{j} & =\frac{R}{2}|V|^{2}-\alpha\langle\nabla \varphi, V\rangle^{2} \\
& \geq \frac{R}{2}|V|^{2}-\frac{\alpha}{2}|\nabla \varphi|^{2}|V|^{2} \\
& =\frac{1}{2} S|V|^{2}
\end{aligned}
$$

which shows that $S_{i j} \geq \frac{1}{2} S g_{i j}$ and the consequence (ii) follows. We complete the proof of Corollary 1.5 .

\section{Proof of Theorem 1.6-Theorem 1.8}

We first prove Theorem 1.6.

Proof of Theorem 1.6. When $n=2$, we have $R_{i j}=\frac{R}{2} g_{i j}$ and

$$
S_{i j}=\frac{R}{2} g_{i j}-\alpha \varphi_{i} \varphi_{j}=\frac{1}{2}\left(S+\alpha|\nabla \varphi|^{2}\right) g_{i j}-\alpha \varphi_{i} \varphi_{j}
$$

Hence,

$$
\begin{aligned}
\left|S_{i j}\right|^{2} & =\frac{R^{2}}{2}+\alpha^{2}|\nabla \varphi|^{4}-\alpha R|\nabla \varphi|^{2} \\
& =\frac{1}{2} S^{2}+\frac{1}{2} \alpha^{2}|\nabla \varphi|^{4}
\end{aligned}
$$

and (2.20) becomes

$$
\begin{aligned}
\frac{d}{d t} \lambda= & -r \lambda+\int_{M}\left\{2 b\left|S_{i j}\right|^{2} u^{2}+2 S^{i j} u_{i} u_{j}+2 b \alpha(\Delta \varphi)^{2} u^{2}\right. \\
& \left.\left.+(2 b-1) b S^{2} u^{2}-(2 b-1) \lambda S u^{2}+(2 b-1) S|\nabla u|^{2}\right]\right\} d v \\
= & -r \lambda+\int_{M}\left\{b S^{2} u^{2}+b \alpha^{2}|\nabla \varphi|^{4} u^{2}+S|\nabla u|^{2}+\alpha|\nabla \varphi|^{2}|\nabla u|^{2}-2 \alpha\langle\nabla u, \nabla \varphi\rangle^{2}\right. \\
& \left.\left.+2 b \alpha(\Delta \varphi)^{2} u^{2}+(2 b-1) b S^{2} u^{2}-(2 b-1) \lambda S u^{2}+(2 b-1) S|\nabla u|^{2}\right]\right\} d v \\
= & -r \lambda+\int_{M}\left\{2 b^{2} S^{2} u^{2}-(2 b-1) \lambda S u^{2}+2 b S|\nabla u|^{2}\right. \\
& \left.\left.+b \alpha^{2}|\nabla \varphi|^{4} u^{2}+\alpha|\nabla \varphi|^{2}|\nabla u|^{2}-2 \alpha\langle\nabla u, \nabla \varphi\rangle^{2}+2 b \alpha(\Delta \varphi)^{2} u^{2}\right]\right\} d v .
\end{aligned}
$$

Therefore, we obtain Theorem 1.6

Proof of Theorem 1.7. In particular, when $r=0$, the rescaled List's extended Ricci flow (1.3) becomes the List's extended Ricci flow (1.2) and 
(3.3) becomes

$$
\begin{aligned}
\frac{d}{d t} \lambda= & \int_{M}\left\{2 b^{2} S^{2} u^{2}+(1-2 b) \lambda S u^{2}+2 b S|\nabla u|^{2}\right. \\
& \left.\left.+b \alpha^{2}|\nabla \varphi|^{4} u^{2}+\alpha|\nabla \varphi|^{2}|\nabla u|^{2}-2 \alpha\langle\nabla u, \nabla \varphi\rangle^{2}+2 b \alpha(\Delta \varphi)^{2} u^{2}\right]\right\} d v
\end{aligned}
$$

respectively. In particular, for $r=0$, we have from (2.24)

$$
S(x, t) \geq \frac{S_{\min }(0)}{1-t S_{\min }(0)} .
$$

Therefore, (3.4) gives

$$
\begin{aligned}
\frac{d}{d t} \lambda & \geq \int_{M}\left\{2 b^{2} S^{2} u^{2}+(1-2 b) \lambda S u^{2}+2 b S|\nabla u|^{2}\right\} d v \\
& \geq \int_{M}\left\{2 b^{2} S^{2} u^{2}+(1-2 b) \lambda S u^{2}+\frac{2 b S_{\min }(0)}{1-t S_{\min }(0)}|\nabla u|^{2}\right\} \\
& =\int_{M}\left\{2 b^{2} S^{2} u^{2}+(1-2 b) \lambda S u^{2}+\frac{2 b S_{\min }(0)}{1-t S_{\min }(0)}\left(\lambda u^{2}-b S u^{2}\right)\right\} \\
& \geq \frac{S_{\min }(0)}{1-t S_{\min }(0)} \lambda+2 b^{2} \int_{M}\left(S^{2}-S \frac{S_{\min }(0)}{1-t S_{\min }(0)}\right) u^{2} d v
\end{aligned}
$$

Using the inequality $y^{2}-c y \geq-\frac{1}{4} c^{2}$ into (3.6), we derive

$$
\frac{d}{d t} \lambda \geq \frac{S_{\min }(0)}{1-t S_{\min }(0)} \lambda-\frac{b^{2} S_{\min }^{2}(0)}{2\left[1-t S_{\min }(0)\right]^{2}}
$$

which shows

$$
\frac{d}{d t}\left\{\left[1-t S_{\min }(0)\right] \lambda-\frac{b^{2} S_{\min }(0)}{2} \ln \left[1-t S_{\min }(0)\right]\right\} \geq 0 .
$$

Integrating both sides of (3.8) on $t$, we have

$$
\lambda(t) \geq \frac{1}{1-t S_{\min }(0)} \lambda(0)+\frac{b^{2} S_{\min }(0)}{2\left[1-t S_{\min }(0)\right]} \ln \left[1-t S_{\min }(0)\right]
$$

and Theorem 1.7 follows.

Proof of Theorem 1.8, Note that (3.3) becomes

$$
\begin{aligned}
\frac{d}{d t} \lambda= & -r \lambda+\int_{M}\left\{2 b^{2} S^{2} u^{2}+(1-2 b) \lambda S u^{2}+2 b S|\nabla u|^{2}\right. \\
& \left.\left.+b \alpha^{2}|\nabla \varphi|^{4} u^{2}+\alpha|\nabla \varphi|^{2}|\nabla u|^{2}-2 \alpha\langle\nabla u, \nabla \varphi\rangle^{2}+2 b \alpha(\Delta \varphi)^{2} u^{2}\right]\right\} d v .
\end{aligned}
$$


In particular, for $n=2$, we have from (2.24)

$$
S(x, t) \geq x(t):=\frac{S_{\min }(0) e^{-\int_{0}^{t} r(s) d s}}{1-S_{\min }(0) \int_{0}^{t}\left(e^{-\int_{0}^{s} r(\tilde{s}) d \tilde{s}}\right) d s} .
$$

Hence, (3.10) yields

$$
\begin{aligned}
\frac{d}{d t} \lambda & \geq-r \lambda+\int_{M}\left\{2 b^{2} S^{2} u^{2}+(1-2 b) \lambda S u^{2}+2 b S|\nabla u|^{2}\right\} d v \\
& \geq-r \lambda+2 b^{2} \int_{M} S^{2} u^{2} d v+(1-2 b) \lambda x(t)+2 b x(t) \int_{M}|\nabla u|^{2} d v \\
& =-r \lambda+2 b^{2} \int_{M} S^{2} u^{2} d v+(1-2 b) \lambda x(t)+2 b x(t)\left\{\lambda-b \int_{M} S u^{2} d v\right\} \\
& =(x(t)-r) \lambda+2 b^{2} \int_{M}(S-x(t)) S u^{2} d v \\
& \geq(x(t)-r) \lambda .
\end{aligned}
$$

Note that $\lambda(t)>0$. We obtain from (3.12)

$$
\frac{d}{d t}(\ln \lambda) \geq x(t)-r .
$$

We complete the proof of Theorem 1.8 .

\section{Proof of Theorem 1.9. Theorem 1.12}

In order to derive our results, we first prove the following three lemmas:

Lemma 4.1. Under the evolution equation (1.23), we have

$$
\begin{gathered}
\left(e^{-f} d v\right)_{t}=\left(-f_{t}-S+r\right) e^{-f} d v=-\left(\Delta e^{-f}\right) d v \\
\left(|\nabla f|^{2}\right)_{t}=2 S_{i j} f^{i} f^{j}-\frac{2 r}{n}|\nabla f|^{2}+2 \nabla f \nabla\left(-\Delta f+|\nabla f|^{2}-S+r\right) .
\end{gathered}
$$

Proof. The formula (4.1) is a direct conclusion of (2.2) in Lemma 2.1 and $f_{t}=-\Delta f+|\nabla f|^{2}-S+r$. Similarly, we have

$$
\begin{aligned}
\left(|\nabla f|^{2}\right)_{t} & =\left(g^{i j} f_{i} f_{j}\right)_{t} \\
& =f_{i} f_{j} \frac{\partial}{\partial t} g^{i j}+2 g^{i j}\left(f_{t}\right)_{i} f_{j} \\
& =2 S^{i j} f_{i} f_{j}-\frac{2 r}{n}|\nabla f|^{2}+2 \nabla f \nabla\left(-\Delta f+|\nabla f|^{2}-S+r\right)
\end{aligned}
$$

and (4.2) is achieved. We complete the proof of Lemma 4.1 . 
Lemma 4.2. With the help of Lemma 4.1, we have

$$
\begin{gathered}
\left(\int_{M} S e^{-f} d v\right)_{t}=\int_{M}\left(2\left|S_{i j}\right|^{2}-\frac{2 r}{n} S+2 \alpha(\Delta \varphi)^{2}\right) e^{-f} d v \\
\left(\int_{M}|\nabla f|^{2} e^{-f} d v\right)_{t}=\int_{M}\left(2 S_{i j} f^{i} f^{j}-\frac{2 r}{n}|\nabla f|^{2}-2 \Delta^{2} f-2 \Delta S+\Delta|\nabla f|^{2}\right) e^{-f} d v \\
\left(\int_{M}\left(|\nabla f|^{2}+S\right) e^{-f} d v\right)_{t}=\int_{M}\left(2\left|S_{i j}\right|^{2}-\frac{2 r}{n}\left(S+|\nabla f|^{2}\right)+2 S_{i j} f^{i} f^{j}\right. \\
\left.-2 \Delta^{2} f-2 \Delta S+\Delta|\nabla f|^{2}+2 \alpha(\Delta \varphi)^{2}\right) e^{-f} d v
\end{gathered}
$$

Proof. By the Stoke formula, we can obtain (4.4) from (2.3) in Lemma 2.1. It is easy to see that (4.5) holds from (4.2). Thus, the formula (4.6) follows by combining (4.4) with (4.5).

Lemma 4.3. Under the rescaled List's extended Ricci flow (1.3), we have

$$
\begin{gathered}
\int_{M}\left|f_{i j}\right|^{2} e^{-f} d v=\int_{M}\left(\frac{1}{2} \Delta|\nabla f|^{2}-\Delta^{2} f-S_{i j} f^{i} f^{j}-\alpha\langle\nabla f, \nabla \varphi\rangle^{2}\right) e^{-f} d v \\
2 \int_{M} S^{i j} f_{i j} e^{-f} d v=\int_{M}\left[2 S_{i j} f^{i} f^{j}-\Delta S+2 \alpha(\Delta \varphi)\langle\nabla f, \nabla \varphi\rangle\right] e^{-f} d v \\
\int_{M}\left|S_{i j}+f_{i j}\right|^{2} e^{-f} d v=\int_{M}\left[\left|S_{i j}\right|^{2}+S_{i j} f^{i} f^{j}+\frac{1}{2} \Delta|\nabla f|^{2}-\Delta^{2} f-\Delta S\right. \\
\left.-\alpha\langle\nabla f, \nabla \varphi\rangle^{2}+2 \alpha(\Delta \varphi)\langle\nabla f, \nabla \varphi\rangle\right] e^{-f} d v .
\end{gathered}
$$

Proof. Multiplying both sides of the following well-known Bochner formula with $e^{-f}$

$$
\frac{1}{2} \Delta|\nabla f|^{2}=\left|f_{i j}\right|^{2}+2 \nabla f \nabla \Delta f+R_{i j} f^{i} f^{j}
$$

and integrating on it, we have

$$
\begin{aligned}
\int_{M}\left|f_{i j}\right|^{2} e^{-f} d v & =\int_{M}\left(\frac{1}{2} \Delta|\nabla f|^{2}-\Delta^{2} f-R_{i j} f^{i} f^{j}\right) e^{-f} d v \\
& =\int_{M}\left(\frac{1}{2} \Delta|\nabla f|^{2}-\Delta^{2} f-S_{i j} f^{i} f^{j}-\alpha\langle\nabla f, \nabla \varphi\rangle^{2}\right) e^{-f} d v
\end{aligned}
$$


and (4.7) follows. On the other hand,

$$
\begin{aligned}
2 \int_{M} S_{i j} f^{i} f^{j} e^{-f} d v & =2 \int_{M}\left(S^{i j} f_{i}\right)_{, j} e^{-f} d v \\
& =2 \int_{M}\left(S^{i j}{ }_{, j} f_{i}+S^{i j} f_{i j}\right) e^{-f} d v .
\end{aligned}
$$

Applying (4.7) into (4.12) gives

$$
\begin{aligned}
2 \int_{M} S^{i j} f_{i} f_{j} e^{-f} d v & =2 \int_{M}\left(\frac{1}{2} S_{, i} f^{i}-\alpha(\Delta \varphi) \varphi_{i} f^{i}+S^{i j} f_{i j}\right) e^{-f} d v \\
& =\int_{M}\left[\Delta S-2 \alpha(\Delta \varphi)\langle\nabla f, \nabla \varphi\rangle+2 S^{i j} f_{i j}\right] e^{-f} d v
\end{aligned}
$$

which gives the desired formula (4.8).

By combining (4.7) with (4.8), we derive (4.9) finally.

Proof of Theorem 1.9. Applying(4.9) into (4.6), we obtain

$$
\begin{aligned}
\frac{d}{d t} \mathcal{F}= & \frac{d}{d t} \int_{M}\left(|\nabla f|^{2}+S\right) e^{-f} d v \\
= & \int_{M}\left(2\left|S_{i j}\right|^{2}-\frac{2 r}{n}\left(S+|\nabla f|^{2}\right)+2 S^{i j} f_{i} f_{j}\right. \\
& \left.-2 \Delta^{2} f-2 \Delta S+\Delta|\nabla f|^{2}+2 \alpha(\Delta \varphi)^{2}\right) e^{-f} d v \\
= & \int_{M}\left(-\frac{2 r}{n}\left(|\nabla f|^{2}+S\right)+2\left|S_{i j}+f_{i j}\right|^{2}\right. \\
& \left.+2 \alpha(\Delta \varphi)^{2}+2 \alpha\langle\nabla f, \nabla \varphi\rangle^{2}-4 \alpha(\Delta \varphi)\langle\nabla f, \nabla \varphi\rangle\right) e^{-f} d v \\
= & -\frac{2 r}{n} \mathcal{F}+2 \int_{M}\left(\left|S_{i j}+f_{i j}\right|^{2}+\alpha|\Delta \varphi-\langle\nabla f, \nabla \varphi\rangle|^{2}\right) e^{-f} d v .
\end{aligned}
$$

Therefore, we obtain

$$
\begin{aligned}
\frac{d}{d t} \mathcal{F}_{k}= & \frac{d}{d t} \mathcal{F}+(k-1) \frac{d}{d t} \int_{M} S e^{-f} d v \\
= & -\frac{2 r}{n} \mathcal{F}_{k}+2(k-1) \int_{M}\left(\left|S_{i j}\right|^{2}+\alpha(\Delta \varphi)^{2}\right) e^{-f} d v \\
& +2 \int_{M}\left(\left|S_{i j}+f_{i j}\right|^{2}+\alpha|\Delta \varphi-\langle\nabla f, \nabla \varphi\rangle|^{2}\right) e^{-f} d v
\end{aligned}
$$

and the desired formula (1.24) is achieved. 
The formula (1.25) can be achieved by a direct computation.

Proof of Theorem 1.10. In order to prove Theorem 1.10, we fist give the following key proposition:

Proposition 4.4. Let $\left(M^{n}, g(t)\right)$ be a compact Riemannian manifold with $g(t)$ satisfying the rescaled List's extended Ricci flow (1.3). We let $\lambda(t)$ be the lowest eigenvalue of the operator $-4 \Delta+k S$ with $k \geq 1$. If there exists a function $v=v(x, t)$ such that

$$
r(t)=\frac{\int_{M}\left(|\nabla v|^{2}+k S\right) e^{-v} d v}{k \int_{M} e^{-v} d v}
$$

then the lowest eigenvalue eigenvalue $\lambda(t)$ is nondecreasing under (1.3) provided $r(t) \leq 0$. The monotonicity is strict unless the metric is Einstein.

Proof. Since the lowest eigenvalue of the operator $-4 \Delta+k S$ on a compact Riemannian manifold is given by

$$
\lambda(g(t))=\inf _{f} \int_{M} \mathcal{F}_{k}(g, f) e^{-f} d v,
$$

where the infimum is taken over functions satisfying $\int_{M} e^{-f} d v=1$. For the compact Riemannian manifold, the lowest eigenvalue $\lambda$ can be attained by a smooth function $u$. Therefore, there exists a smooth function $u$ such that $(-4 \Delta+k S) u=\lambda u$ and

$$
\lambda(g(t))=\int_{M}\left(4|\nabla u|^{2}+k S u^{2}\right) d v=\int_{M}\left(|\nabla \tilde{f}|^{2}+k S\right) e^{-\tilde{f}} d v
$$

by letting $-\tilde{f}=2 \ln u$. Applying (4.18) into (1.25) gives

$$
\begin{aligned}
\frac{d}{d t} \lambda= & \frac{2 r}{n}(\lambda-k r)+2(k-1) \int_{M}\left(\left|S_{i j}-\frac{r}{n} g_{i j}\right|^{2}+\alpha(\Delta \varphi)^{2}\right) e^{-\tilde{f}} d v \\
& +2 \int_{M}\left(\left|S_{i j}+\tilde{f}_{i j}-\frac{r}{n} g_{i j}\right|^{2}+\alpha|\Delta \varphi-\langle\nabla \tilde{f}, \nabla \varphi\rangle|^{2}\right) e^{-\tilde{f}} d v .
\end{aligned}
$$

By using the assumption (4.16), we get

$$
\begin{aligned}
k r & =\int_{M}\left(|\nabla v|^{2}+k S\right) e^{-v} d v \\
& \geq \int_{M}\left(|\nabla \tilde{f}|^{2}+k S\right) e^{-\tilde{f}} d v \\
& =\lambda
\end{aligned}
$$

which means that $\lambda-k r \leq 0$. Therefore, if $r(t) \leq 0$, then (4.19) shows that $\lambda(t)$ is nondecreasing under (1.3). The monotonicity is strict unless

$$
S_{i j}-\frac{r}{n} g_{i j}=0, \quad \Delta \varphi=0
$$


and

$$
S_{i j}+\tilde{f}_{i j}-\frac{r}{n} g_{i j}=0, \quad \Delta \varphi-\langle\nabla \tilde{f}, \nabla \varphi\rangle=0 .
$$

Notice that the Riemannian manifold is compact. Hence, we have $\varphi$ is constant from the second equality in (4.21) or the second equality in (4.22). Therefore, the metric $g$ is Einstein. We complete the proof of Proposition 4.4 .

Now, we are in a position to prove Theorem 1.10. We note that for the extended Hamilton normalized flow under the List's extended Ricci flow, we have

$$
r(t)=\frac{\int_{M} S d v}{\int_{M} d v} .
$$

Choosing $v=\ln \left(\operatorname{Vol}\left(M^{n}\right)\right)$ in (4.16), we derive Theorem 1.10,

Proof of Theorem 1.12. Since the $\mathcal{W}$-functional is related with $\mathcal{F}$ by

$$
\begin{aligned}
\mathcal{W} & =\tau^{2} \int_{M}\left(S+\frac{n}{2 \tau}+|\nabla f|^{2}\right) e^{-f} d v \\
& =\tau^{2} \mathcal{F}+\frac{n \tau}{2},
\end{aligned}
$$

with the help of (1.24), we obtain

$$
\begin{aligned}
\frac{d}{d t} \mathcal{W}= & \tau^{2} \frac{d}{d t} \mathcal{F}+2 \tau \mathcal{F}+\frac{n}{2} \\
= & \tau^{2}\left\{-\frac{2 r}{n} \mathcal{F}+2 \int_{M}\left(\left|S_{i j}+f_{i j}\right|^{2}+\alpha|\Delta \varphi-\langle\nabla f, \nabla \varphi\rangle|^{2}\right) e^{-f} d v\right\} \\
& +2 \tau \mathcal{F}+\frac{n}{2} .
\end{aligned}
$$

Applying

$$
2 \tau^{2} \int_{M}\left|S_{i j}+f_{i j}+\frac{1}{2 \tau} g_{i j}\right|^{2} e^{-f} d v=2 \tau^{2} \int_{M}\left|S_{i j}+f_{i j}\right|^{2} e^{-f} d v+2 \tau \mathcal{F}+\frac{n}{2}
$$

into (4.24) yields

$$
\begin{aligned}
\frac{d}{d t} \mathcal{W}= & 2 \tau^{2}\left\{-\frac{r}{n} \mathcal{F}+\int_{M}\left(\left|S_{i j}+f_{i j}+\frac{1}{2 \tau} g_{i j}\right|^{2}\right.\right. \\
& \left.\left.+\alpha|\Delta \varphi-\langle\nabla f, \nabla \varphi\rangle|^{2}\right) e^{-f} d v\right\} .
\end{aligned}
$$

By the definition of $\mathcal{W}_{k}$, we know

$$
\mathcal{W}_{k}=\mathcal{W}+(k-1) \tau^{2} \int_{M} S e^{-f} d v+\frac{(k-1) n \tau}{2} .
$$


Thus, we get from (4.4) and (4.25)

$$
\begin{aligned}
\frac{d}{d t} \mathcal{W}_{k}= & \frac{d}{d t} \mathcal{W}+\frac{(k-1) n}{2}+2(k-1) \tau \int_{M} S e^{-f} d v \\
& +(k-1) \tau^{2}\left(\int_{M} S e^{-f} d v\right)_{t} \\
= & 2 \tau^{2}\left\{-\frac{r}{n} \mathcal{F}+\int_{M}\left(\left|S_{i j}+f_{i j}+\frac{1}{2 \tau} g_{i j}\right|^{2}\right.\right. \\
& \left.\left.+\alpha|\Delta \varphi-\langle\nabla f, \nabla \varphi\rangle|^{2}\right) e^{-f} d v\right\}+\frac{(k-1) n}{2}+2(k-1) \tau \int_{M} S e^{-f} d v \\
& +(k-1) \tau^{2} \int_{M}\left(2\left|S_{i j}\right|^{2}-\frac{2 r}{n} S+2 \alpha(\Delta \varphi)^{2}\right) e^{-f} d v \\
= & 2 \tau^{2}\left\{-\frac{r}{n} \mathcal{F}_{k}+(k-1) \int_{M}\left(\left|S_{i j}+\frac{1}{2 \tau} g_{i j}\right|^{2}+\alpha(\Delta \varphi)^{2}\right) e^{-f} d v\right. \\
& \left.+\int_{M}\left(\left|S_{i j}+f_{i j}+\frac{1}{2 \tau} g_{i j}\right|^{2}+\alpha|\Delta \varphi-\langle\nabla f, \nabla \varphi\rangle|^{2}\right) e^{-f} d v\right\},
\end{aligned}
$$

and the desired formula (1.29) is attained.

The formula (1.30) can be achieved by a direct computation.

\section{REFERENCES}

[1] X.D. Cao, Xiaodong Eigenvalues of $\left(-\Delta+\frac{R}{2}\right)$ on manifolds with nonnegative curvature operator, Math. Ann. 337 (2007), 435-441.

[2] X.D. Cao, First eigenvalues of geometric operators under the Ricci flow, Proc. Amer. Math. Soc. 136 (2008), 4075-4078.

[3] X.D. Cao, S.B. Hou, J. Ling, Estimate and monotonicity of the first eigenvalue under the Ricci flow, Math. Ann. 354 (2012) 451-463.

[4] B. Chow, P. Lu, L. Ni, Hamilton's Ricci Flow, Graduate Studies in Mathematics, 77. American Mathematical Society, Providence, RI; Science Press, New York, 2006.

[5] S.W. Fang, H.F. Xu, P. Zhu, Evolution and monotonicity of eigenvalues under the Ricci flow, Sci. China Math. 58 (2015), 1737-1744.

[6] B. List, Evolution of an extended Ricci flow system, PhD Thesis. AEI Potsdam. http://www.diss.fu-berlin.de/2006/180/index.html (2006).

[7] B. List, Evolution of an extended Ricci flow system, Comm. Anal. Geom. 16(2008), 1007-1048.

[8] Y. Li, Eigenvalues and entropies under the harmonic-Ricci flow, Pacific J. Math. 267 (2014), 141-184.

[9] J.F. Li, Evolution of eigenvalues along rescaled Ricci flow, Canad. Math. Bull. 56 (2013), 127-135.

[10] L. Ma, Eigenvalue monotonicity for the Ricci-Hamilton flow, Ann. Global Anal. Geom. 29 (2006), 287-292. 
[11] R. Müller, Ricci flow coupled with harmonic map flow, Ann. Sci. Éc. Norm. Supér. 45 (2012), 101-142.

College of Mathematics and Information Science, Henan Normal UniverSity, Xinxiang, Henan 453007, People's Republic of China

E-mail address: hgy@henannu.edu.cn

College of Mathematics and Information Science, Henan Normal UniverSity, Xinxiang, Henan 453007, People's Republic of China

E-mail address: lizhihnsd@126.com 